\title{
Decision Support System for Selecting Collaborative Product Development Partners
}

\author{
Masataka Yoshimura, ${ }^{1, *}$ Kazuhiro Izui ${ }^{1}$ and Shusaku Kida ${ }^{2}$ \\ ${ }^{1}$ Department of Precision Engineering, Graduate School of Engineering, Kyoto University, Kyoto, Japan \\ ${ }^{2}$ Graduate School of Information Science, Nara Institute of Science and Technology, Nara, Japan
}

\begin{abstract}
Collaboration is becoming a new current paradigm for product manufacturing, as computer networks facilitate collaboration with partners located in widely distributed locations. Computer network technologies allow a large number of candidate partners to be examined for possible collaboration, so that the most suitable partner, or partners, can be selected from a broader, more diverse group than previously possible. In order to take best advantage of the collaboration paradigm, the precise method for selecting collaborative product development partners is an important technological point. Failed multicompany collaboration projects can do serious harm to the member companies on a number of fronts, in terms of financial cost, loss of prestige, loss of market share, and so on. The optimal collaboration partners should be selected from a group of candidates, so that production of new products can be achieved at a minimum cost, both financial and in terms of effort and expended resources. This paper proposes a decision supporting method for selecting an optimum collaborative product development partner from a group of potential partners. First, the effectiveness of collaborative product development and the need for a partner selection method is clarified. Next, a method for selecting the most suitable product development partner is constructed. Here, technologies that are required for developing the new product are classified into two groups: (1) technologies that have already been developed, and (2) technologies that must be newly developed. The proposed method first excludes unsuitable candidate partners, based on their achievement level concerning existing required technologies, and then selects the most suitable partner from the standpoint of technologies that must be newly developed. Finally, a case study is given to demonstrate the utility of the proposed method.
\end{abstract}

Key Words: collaboration, collaboration partner, product development, knowledge sharing, technological sharing, decision making, partner benefit evaluation, partner selection, computer network.

\section{Introduction}

Collaboration means that participants having different knowledge and technologies generate new concrete ideas and products by virtue of their cooperative effort.

The main features of such collaboration are:

(1) An enlarged range of available knowledge and technologies.

(2) Enhanced possibility for superior solutions to design problems, due to mutual creative stimulation of participants during the idea generating and refinement processes.

Customers are always searching for new and attractive products. Companies are therefore continually forced to improve existing products and prepare new necessary technologies, to bring fresh, desirable

*Author to whom correspondence should be addressed.

E-mail: yoshimura@prec.kyoto-u.ac.jp products to the market. In such circumstances, more effective and timely product designs can be achieved when product development is collaboratively conducted with partners that have already developed necessary technologies that the company initiating the project lacks.

Research related to the subject of this paper falls under two main categories, that dealing with collaborative and cooperative design problems $[9,10,12]$, and that which focuses on effectively realizing the benefits of concurrent engineering [6,7]. Collaboration among different divisions, different enterprises, or groups of engineers possessing diverse knowledge, is considered to be one of the most promising methods for stimulating advanced product design and improving manufacturing performance [14]. Yoshimura and Yoshikawa discussed a system supporting collaboration, where different groups and/or enterprises having competitive relationships consider collaborative projects [16]. Certain collaborative product design integration methods that can be used by experts, utilizing mobile agents operating in networked computing environments, have also been presented $[1,13,15]$. 
Collaboration during product design and manufacturing is closely related with project management activities, and this has been examined for multinational environments [4]. Factors influencing the utility of communication in distributed product development environments were discussed [11], and the effects of information sharing on supply chain performances were examined with respect to electronic commerce [3]. Prasad et al. discussed a systematic concurrent workflow management consisting of planning and scheduling teams' activities, to support cooperative and concurrent projects [8]. Most recently, project management activities that improve design process cost performance were examined [2].

Computer networks make collaborating with partners located in isolated locations quite easy. Utilization of computer network technologies also facilitates examining a large number of candidate partners for possible collaboration, so that the most suitable partner can be selected from among as broad a collection of likely candidate partners as feasible. The ultimate success of collaboration critically depends on the precise selection of the most appropriate collaboration partner. Despite the potential advantages offered, however, methods for selecting optimum collaboration partners using quantitative logical procedures have seldom been studied.

This paper proposes a decision supporting method for selecting the optimum collaborative product development partner from a group of potential partners. In Section 2, a method for selecting the most suitable product development partner is constructed and explained in detail. Then, in Section 3, case studies are given to demonstrate the effectiveness of the proposed method.

\section{Methodologies for the Selection of a Product Development Partner}

\subsection{Description of the Problem}

Assume that a primary company is considering development of a new product that requires collaboration with a suitable partner company, since it does not have at its command all the technologies required for developing the product, as shown in Figure 1. A method for selecting the most suitable product development partner from among a group of candidate partners is constructed. Technologies that are required for developing the new product are here classified into two groups: (1) technologies that have already been developed and (2) technologies that must be newly developed.

The number of existing technologies used for developing a product is denoted $n$. The $j$ th existing technology is expressed as $\alpha_{j}$. The set of existing

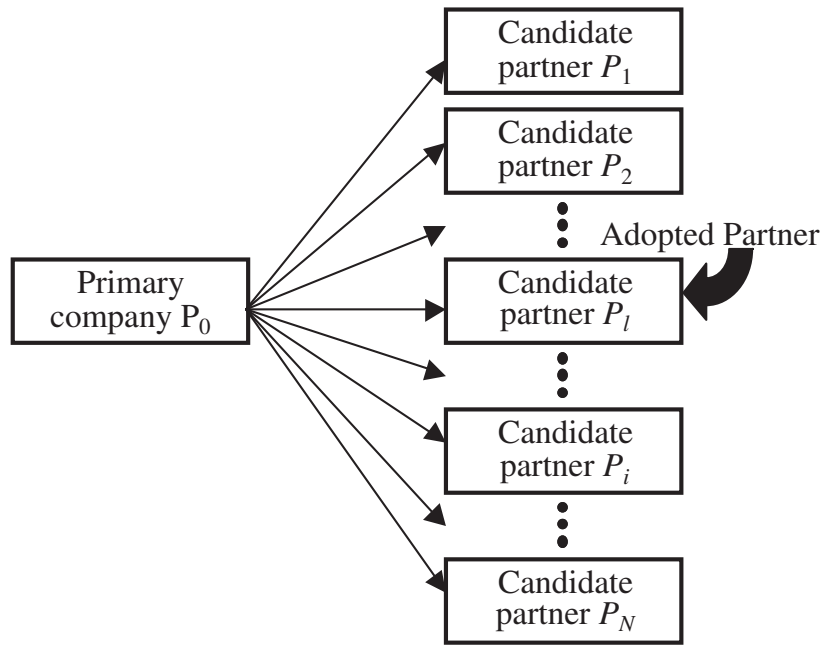

Figure 1. Conceptual diagram of collaboration with a partner company.

technologies, $A$, is denoted $A=\left\{\alpha_{1}, \alpha_{2}, \ldots, \alpha_{j}, \ldots, \alpha_{n}\right\}$. The number of necessary technologies to be newly developed is denoted $m$. The $k$ th technology to be newly developed is expressed as $\gamma_{k}$. The set of technologies to be newly developed, $G$, is denoted $G=\left\{\gamma_{1}, \gamma_{2}, \ldots\right.$, $\left.\gamma_{k}, \ldots, \gamma_{m}\right\}$.

Candidate partners are denoted $P_{1}, P_{2}, \ldots, P_{i}, \ldots, P_{N}$. The total number of candidate partners is $N$. The proposed method first excludes unsuitable candidate partners, based on their level of existing technologies, and then selects the most suitable partner from the standpoint of technologies that must be newly developed.

\subsection{Existing Technologies}

\subsubsection{WEIGHTING OF THE IMPORTANCE OF EXISTING TECHNOLOGIES}

The importance levels of existing technologies usually depend on requirement levels in the market, as manifested by the conditions concerning product demand, the presence of competitors, the maturity of the technology, and so on. The importance level for existing technology $\alpha_{j}$ is denoted $\omega_{j}$, which is determined by the market value of the technology.

\subsubsection{EVALUATION OF TECHNOLOGIES}

The technological level of the primary company and candidate partners for existing technology $\alpha_{j}$ is evaluated using a cost measure $C_{j}$ for the representative technological characteristic level $t_{j}$. First, the relationship between $t_{j}$ and $C_{j}$ is graphically displayed, as shown in Figure 2. The highest technological level in the particular industrial field corresponds to point A, while the average technological level is indicated by point B. The ordinate axis represents the cost on a dimensionless scale from 0 to 10 , not as a monetary amount. A cost 


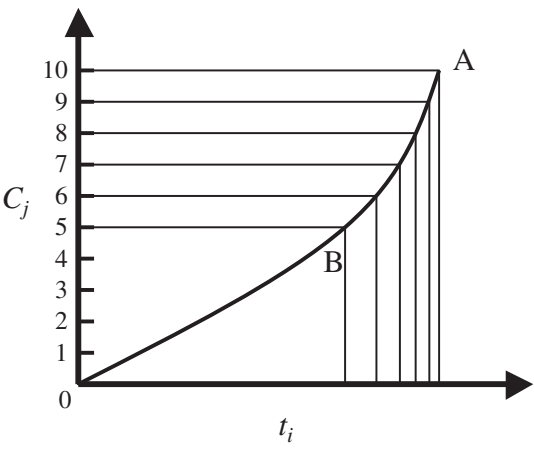

Figure 2. Relationship between representative technological characteristics and cost measures.

value of 10 on the vertical axis of Figure 2 is assigned to the technological level at point $\mathrm{A}$, while a cost value of 5 is assigned to the technological level at point B. In the Figure 2 graph, both the ordinate and abscissa have linear scales. The levels of existing technologies are evaluated according to their corresponding costs, which range in value from 0 to 10 .

\subsubsection{DETERMINING THE EXTENT OF SHARED TECHNOLOGIES}

When selecting a partner for collaboration, it should, as much as possible, be chosen for its expertise in technologies that are different from those held by the primary company initiating the cooperative project. The absolute value $\theta_{j P_{0} P_{i}}$ of the difference between the evaluation level for technology $j$ offered by candidate partner $P_{i}$ and the evaluation level for technology $j$ owned by primary company $P_{0}$ is denoted:

$$
\theta_{j P_{0} P_{i}}=\left|T_{j P_{0}}-T_{j P_{i}}\right|
$$

where, $T_{j P_{0}}$ is the evaluation level of primary company $P_{0}$ 's technology $\alpha_{j}$ and $T_{j P_{i}}$ is the evaluation level of candidate partner $P_{j}^{\prime}$ 's technology $\alpha_{j}$.

The sum $S_{i}$ of the absolute values of the evaluation level differences in technologies where primary company $P_{0}$ 's technology is lower than candidate partner $P_{i}$ 's technology level is obtained under the condition of $\theta_{j P_{0} P_{i}}=0$ when $T_{j P_{0}}<T_{j P_{i}}$ as follows:

$$
S_{i}=\sum \omega_{j} \theta_{j P_{0} P_{i}}
$$

The sum $I_{i}$ of the absolute values of the evaluation level differences in technologies where candidate partner $P_{i}$ 's technology level is lower than primary company $P_{0}$ 's technology level is obtained under the condition of $\theta_{j P_{0} P_{i}}=0$ when $T_{j P_{0}}>T_{j P_{i}}$ as follows:

$$
I_{i}=\sum \omega_{j} \theta_{j P_{0} P_{i}}
$$

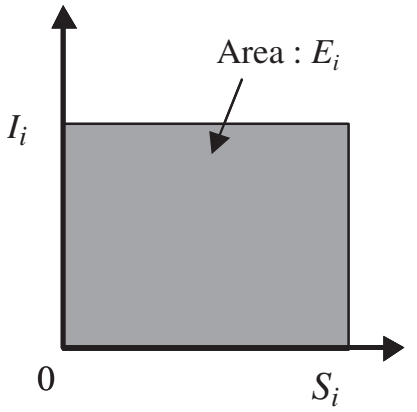

Figure 3. Relationship $S_{i}$ and $I_{i}$ (absolute values of technological level differences for superior and inferior evaluation vis-à-vis the primary company).

Then, the value $E_{i}$ of the existing technologies that the primary and partner company pair can expect to use without further development during collaboration is expressed as:

$$
E_{i}=S_{i} \times I_{i}
$$

The value of $E_{i}$ also represents the magnitude of the differences in the specialties of the primary and partner company technologies. Greater differences yield larger values of $E_{i}$. When $S_{i}$ and $I_{i}$ are, respectively, expressed on the abscissa and ordinate of rectangular coordinates as shown in Figure 3, $E_{i}$ corresponds to the area of the rectangular region. The difference levels in the specialties within the primary and partner company technologies can thus be visually understood, and larger areas represent correspondingly greater differences in the levels of technology held by the two companies. Although the larger the graphed area, the greater the benefits of collaboration, the shape of the area is critical. Square shapes indicate a mutually beneficial relationship, where elongated rectangular shapes reveal a fundamental asymmetry in the potential collaboration, where one company alone benefits. Such unbalanced collaborations are likely to result in failure.

\subsection{Evaluation of Candidate Partners Considering Existing Technology}

Unpromising candidate partners are excluded using the following procedures:

(1) The differences between the horizontal and vertical lengths of the rectangular shapes $S_{i}$ and $I_{i}$ shown in Figure 3 are evaluated. Candidate partners showing large differences between $S_{i}$ and $I_{i}$ are excluded from the list of candidate partners, due to the basic asymmetry in the expected benefits.

(2) Then, the magnitudes of $E_{i}$ are compared among the candidate partners. Candidate partners having small magnitudes of $E_{i}$ are excluded from the list of 
candidate partners since minimal benefit would accrue from collaboration.

\subsection{Technologies to be Newly Developed}

\subsubsection{EVALUATING TECHNOLOGIES TO BE NEWLY DEVELOPED}

Evaluation of technologies to be newly developed is conducted using the cost measures corresponding to the representative technological level, in the same way as for evaluations of existing technologies.

The goal level of the technology is 10 , while the mean level of the technology in the industrial field is 3 . The level scale is linear.

\subsubsection{REQUIRED AMOUNT OF TECHNOLOGICAL DEVELOPMENT}

The differences between the goal level of technologies to be developed and the present level of technology are obtained. The required amount of technology $\gamma_{k}$ that must be newly developed, $L_{\gamma_{k} P_{i}}$, is defined for the primary company $P_{0}$ and candidate partner $P_{i}$ as follows:

$$
L_{\gamma_{k}} P_{i}=F_{k g}-F_{k a}
$$

where, $F_{k g}$ is the goal level of technology $\gamma_{k}$ to be developed, and $F_{k a}$ is the present level of technology $\gamma_{k}$.

\subsubsection{SUMMATION OF THE REQUIRED AMOUNT OF TECHNOLOGIES TO BE NEWLY DEVELOPED}

The total amount of development required for the development of a new product without collaboration, $\varphi_{P_{i}}$, is obtained as follows:

$$
\varphi_{P_{i}}=\sum_{k=1}^{m} L_{\gamma_{k} P_{i}}
$$

$\varphi_{P_{i}}$ also expresses the level of developmental difficulty without collaboration for the product under consideration.

The quantitative sum of technologies to be collaboratively developed is denoted $\varphi_{P_{0} P_{i}}$ and is obtained as follows:

$$
\varphi_{P_{0} P_{i}}=\sum_{k=1}^{m} \min \left(L_{\gamma_{k} P_{0}}, L_{\gamma_{k} P_{i}}\right)
$$

\subsubsection{REDUCTION RATIO FOR THE AMOUNT OF TECHNOLOGIES TO BE NEWLY DEVELOPED UNDER COLLABORATION}

The reduction ratio for the amount of technologies to be newly developed under collaboration between the primary company and a candidate partner is calculated using the total amount of development required for the development of a new product.

The following concerns the actual collaboration with a partner. During the collaboration, the partner best able to develop the highest level of technology for the area in question for the least cost is utilized. In most cases, the partner whose new technology requires less additional development is selected.

The ratio representing the reduction in the amount of technologies that must be newly developed when collaboration is conducted, $D_{0 i}$, is defined with respect to the primary company as follows:

$$
D_{0 i}=\frac{\varphi_{P_{0}}-\varphi_{P_{0} P_{i}}}{\varphi_{P_{0}}} \times 100
$$

The reduction ratio in the amount of technologies that must be newly developed when collaboration is conducted, $D_{i}$, is defined with respect to a candidate partner company $P_{i}$ as follows:

$$
D_{i}=\frac{\varphi_{P_{i}}-\varphi_{P_{0} P_{i}}}{\varphi_{P_{i}}} \times 100
$$

\subsection{Evaluation of Candidate Partners Considering Technologies to be Newly Developed}

The following points are considered concerning technologies to be newly developed when selecting the most suitable partner company from the remaining group of candidate partners:

(1) Partners having smaller magnitudes of $\varphi_{P_{0} P_{i}}$ are more preferable.

(2) Values of $D_{0 i}$ and $D_{i}$ must be similar to a degree initially specified by decision makers.

\subsection{Discussion Concerning Procedures for Selecting Product Development Partners}

Existing technologies can be evaluated using existing data, but this is obviously not possible for technologies that must be newly developed. The evaluation of technologies under development includes uncertain aspects such as goal definitions, technical breakthroughs, proprietary research and so on. Hence, when definite benefits capable of being evaluated are required, the number of candidate partners can be greatly reduced by the evaluation of $E_{i}$. When the importance of technologies to be newly developed is foremost, the number of candidate partners remaining after evaluating $E_{i}$ is larger. 
In the proposed method, practical problems are formulated using simplified numerical approaches. Practical problems may include more specific factors, but these could be evaluated by adding further elements to the fundamental procedures proposed in this paper. The proposed quantitative decision making method, though simplified, can nevertheless provide a useful guideline for selecting the most suitable collaborative partners.

\section{Case Study of the Development Process for a Product}

\subsection{Problem Description}

Consider a new product $A$ that is to be produced under collaborative development between an initiating company, here called the primary company, and a partner company. In the following scenario, to create product $A$, there are five existing technologies $\alpha_{1}, \alpha_{2}, \ldots, \alpha_{5}$ while three required technologies, $\gamma_{1}, \gamma_{2}$, and $\gamma_{3}$, need to be newly developed. The primary company possesses all the existing technologies. Table 1 shows the market value for each technology.

Five candidate collaboration partners $P_{1}, P_{2}, \ldots, P_{5}$ are considered. The existing levels of achievement for the various technologies held by the candidate collaboration partners and the primary company are given in Table 2. The technological achievement levels shown in Table 2 are derived from the relationship between technological levels and their corresponding cost measures, as shown in Figure 2.

The primary company has some basic knowledge concerning technologies $\gamma_{1}$ and $\gamma_{2}$ which need to be newly developed, but none for technology $\gamma_{3}$. The development partner is selected from companies having some knowledge concerning technology $\gamma_{3}$.

Table 1. Market value for existing technologies.

\begin{tabular}{llllll}
\hline Existing Technology & $\alpha_{1}$ & $\alpha_{2}$ & $\alpha_{3}$ & $\alpha_{4}$ & $\alpha_{5}$ \\
\hline Market value & 60 & 55 & 55 & 50 & 45 \\
\hline
\end{tabular}

Table 2. Existing technological achievement levels for primary company and candidate collaboration partners.

\begin{tabular}{lccccc}
\hline & $\alpha_{1}$ & $\alpha_{2}$ & $\alpha_{3}$ & $\alpha_{\mathbf{4}}$ & $\alpha_{5}$ \\
\hline Primary company $P_{0}$ & 7 & 7 & 6 & 5 & 4 \\
Candidate partner $P_{1}$ & 9 & 6 & 6 & 0 & 9 \\
Candidate partner $P_{2}$ & 0 & 8 & 6 & 8 & 6 \\
Candidate partner $P_{3}$ & 9 & 8 & 9 & 0 & 0 \\
Candidate partner $P_{4}$ & 8 & 6 & 0 & 8 & 9 \\
Candidate partner $P_{5}$ & 8 & 0 & 7 & 7 & 9 \\
\hline
\end{tabular}

\subsection{Selection of a Collaboration Partner Based on the Amount of Technological Sharing}

The importance levels, or weighting coefficients, for the existing technologies are shown in Table 3. The ratios among the market values for each existing technology given in Table 1 are used when calculating these weighting coefficients.

The values of $S_{i}$ and $I_{i}$, the ratio of $S_{i}$ and $I_{i}$, and the $E_{i}$ value for each partner are shown in Table 4.

It can be seen that the ratio of $S_{i}$ and $I_{i}$ for each partner is close to 1 ; that is, the difference level between $S_{i}$ and $I_{i}$ is small. The ratio of $S_{i}$ and $I_{i}$ for each candidate partner indicates that collaboration might be successful, but candidate partners $P_{1}$ and $P_{2}$ were excluded from the group of possible candidates since their $E_{i}$ values were too low.

\subsection{Required Amount of Technologies to be Newly Developed}

For the technologies $\gamma_{1}, \gamma_{2}$, and $\gamma_{3}$ that must be newly developed, the current levels of technological achievement for $\gamma_{1}, \gamma_{2}$, and $\gamma_{3}$ for each of the remaining candidate partners $P_{3}, P_{4}$, and $P_{5}$, the required amount of technologies to be newly developed, $L_{\gamma_{k} P_{i}}$, and the sum of the required amount of technologies to be newly developed, $\varphi_{P_{i}}$ are shown in Table 5 .

Table 3. Weighting coefficients for existing technologies.

\begin{tabular}{llllll}
\hline Existing Technology & $\alpha_{1}$ & $\alpha_{2}$ & $\alpha_{3}$ & $\alpha_{4}$ & $\alpha_{5}$ \\
\hline Weighting coefficients $\omega_{j}$ & 12 & 11 & 11 & 10 & 9 \\
\hline
\end{tabular}

Table 4. Evaluation values for each candidate partner based on existing technologies.

\begin{tabular}{lccccc}
\hline Candidate Partner $\boldsymbol{P}_{\boldsymbol{i}}$ & $\boldsymbol{P}_{\mathbf{1}}$ & $\boldsymbol{P}_{\mathbf{2}}$ & $\boldsymbol{P}_{\mathbf{3}}$ & $\boldsymbol{P}_{\mathbf{4}}$ & $\boldsymbol{P}_{\mathbf{5}}$ \\
\hline$S_{i}$ & 61 & 84 & 86 & 77 & 77 \\
$I_{i}$ & 69 & 59 & 79 & 96 & 98 \\
$S_{i}: I_{i}$ & $1: 1.1$ & $1.4: 1$ & $1.1: 1$ & $1: 1.2$ & $1: 1.3$ \\
$E_{i}$ & 4209 & 4956 & 6794 & 7392 & 7546 \\
\hline
\end{tabular}

Table 5. Required amount of technologies to be newly developed.

\begin{tabular}{lcccccccc}
\hline & $\gamma_{\mathbf{1}}$ & $\gamma_{\mathbf{2}}$ & $\gamma_{\mathbf{3}}$ & $\boldsymbol{L}_{\gamma_{1} \boldsymbol{P}_{\boldsymbol{i}}}$ & $\boldsymbol{L}_{\gamma_{2} \boldsymbol{P}_{\boldsymbol{i}}}$ & $\boldsymbol{L}_{\gamma_{3} \boldsymbol{P}_{\boldsymbol{i}}}$ & $\varphi_{\boldsymbol{P}_{\boldsymbol{i}}}$ & $\varphi_{\boldsymbol{P}_{\mathbf{0}} \boldsymbol{P}_{\boldsymbol{i}}}$ \\
\hline Primary company $P_{0}$ & 4 & 4 & 0 & 6 & 6 & 10 & 22 & - \\
Candidate partner $P_{3}$ & 0 & 6 & 4 & 10 & 4 & 6 & 20 & 16 \\
Candidate partner $P_{4}$ & 4 & 3 & 5 & 6 & 7 & 5 & 18 & 17 \\
Candidate partner $P_{5}$ & 5 & 0 & 6 & 5 & 10 & 4 & 19 & 15 \\
\hline
\end{tabular}


For collaboration, candidate partners having smaller values of $\varphi_{P_{0} P_{i}}$ are preferable, hence the order of candidate partners in terms of their suitability here is $P_{5}, P_{3}, P_{4}$.

\subsection{The Reduction Ratio for the Amount of Technologies to be Newly Developed}

The utility of the collaboration can be determined by evaluating the reduction in the amount of technologies to be newly developed with respect to the primary company, with the candidate partner included in the design project.

The reduction ratios $D_{05}$ and $D_{5}$ for the amount of technologies to be newly developed, from the point of view of the primary company and the 'best' candidate partner chosen above, respectively, are as follows:

$$
D_{05}=31.8 \%, \quad D_{5}=21.1 \%
$$

The results were considered to be satisfactory, i.e., high enough for $P_{5}$ to be selected as the collaboration partner.

The difference in the required amounts of technologies to be newly developed was judged to be satisfactorily small for both the primary company and the candidate partner.

\section{Conclusions}

A method supporting decision making for selecting an optimum collaborative product development partner was proposed. A case study was given to demonstrate the proposed method. The method was shown to be effective in selecting the optimal collaboration partner from a group of candidates, so that production of a new product could be achieved with a minimum cost, both financial and in terms of effort and expended resources.

The current business climate, where a large number of companies are often compelled to pursue development of similar products, may be deleterious to both the environment and the global economy. Cutthroat competition among companies too often leads to thoughtless destruction of nature and poor utilization of natural resources, and technological escalation between companies, each seeking to outdo the other when competing for customers, does not guarantee socially useful benefits. Hence, collaboration among companies in a global marketplace is a promising production paradigm, now, and for the future. However, failed collaborative efforts among companies are a danger, and the number of corporations experiencing difficulties after mergers or cooperative projects has been increasing. The selection of the most suitable product development partner, using methods such as the one discussed in this paper, is thus an increasingly important technological subject.

\section{References}

1. D'Ambrosio, J., Darr, T. and Birmingham, W. (1996). Hierarchical Concurrent Engineering in Multiagent Framework, Concurrent Engineering: Research and Applications, 4(1): 47-56.

2. Kuprenas, J.A. (2003). Project Management Actions to Improve Design Phase Cost Performance, Journal of Management in Engineering, 19(1): 25-32.

3. Kruglianskas, I. and Thamhain, H.J. (2000). Managing Technology-Based Projects in Multinational Environments, IEEE Transactions on Engineering Management, 47(1): 55-64.

4. Lin, F.-R., Huang, S.-H. and Lin S.-C. (2002). Effects of Information Sharing on Supply Chain Performance in Electronic Commerce, IEEE Transactions on Engineering Management, 49(3): 258-268.

5. Mitchell, W. and Singh, K. (1996). Survival of Businesses using Collaborative Relationships to Commercialize Complex Goods, Strategic Management Journal, 17: 169-195.

6. Prasad, B. (1996). Concurrent Engineering Fundamentals Vol. I - Integrated Product and Process Organization, Upper Saddle River, NJ, Prentice Hall PTR.

7. Prasad, B. (1997). Concurrent Engineering Fundamentals Vol. II - Integrated Product Development, Upper Saddle River, NJ, Prentice Hall PTR.

8. Prasad, B., Wang, F. and Deng, J. (1998). A Concurrent Workflow Management Process for Integrated Product Development, Journal of Engineering Design, 9(2): 121-135.

9. Tappeta, R.V. and Renaud, J.E. (1997). Multiobjective Collaborative Optimization. Journal of Mechanical Design, 119(3): 403-411.

10. Shibao, K. and Naka, Y. (1994). Optimisation of the Distributed System by Autonomous Cooperation, In: Proceedings of DARS 94, Distributed Automation Robotic System, pp. 29-40.

11. Sosa, M.E., Eppinger, S.D., Pich, M., McKenddrick, D.G. and Stout, S.K. (2002). Factors that Influence Technical Communication in Distributed Product Development: An Empirical Study in the Telecommunications Industry, IEEE Transactions on Engineering Management, 49(1): 45-58.

12. Sreeram, R.T. and Chawdhry, P.K. (1998). A Single Function Agent Framework for Task Decomposition and Conflict Negotiation, In: ASME Proceeding of DETC'98/ DFM-5748.

13. Yan, J. and Weihua, Z. (1999). Agent-Based Knowledge Management for Collaborative Engineering, In: $A S M E$ Proceeding of DETC'99/EIM-9022.

14. Yoshimura, M. (2002). Collaborative Optimization for Product Design and Manufacturing, In: Proceedings of CAD2002 - Corporate Engineering Research, Dresden, pp. 1-14.

15. Yoshimura, M. and Takahashi, K. (2001). Collaborative Design among Different Fields in Mobile-agent Environments, International Journal of Concurrent Engineering: Research and Applications, 9(2): 146-154.

16. Yoshimura, M. and Yoshikawa, K. (1998). Synergy Effects of Sharing Knowledge During Cooperative Product Design, Concurrent Engineering: Research and Applications, 6(1): 7-14. 


\section{Masataka Yoshimura}

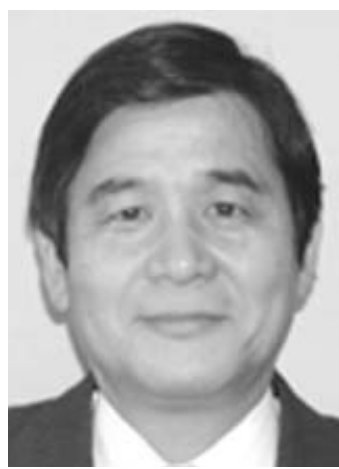

Masataka Yoshimura earned his Bachelor of Engineering Degree in Mechanical Engineering and Master of Engineering Degree in Precision Engineering from Kyoto University, and in 1976, received his Doctor of Engineering Degree from Kyoto University. He is now a Professor in the Graduate School of Engineering at Kyoto University. His research interests include concurrent optimization of product design and manufacturing, information systems for manufacturing, collaborative optimization, concurrent engineering, and dynamics of machine tools and industrial robots. He has published more than 100 English papers in the journals and proceedings of the ASME, AIAA, IJPR, CERA (Concurrent Engineering: Research and Applications), Structural Optimization, the JSME (Japan Society of Mechanical Engineers), and the JSPE (Japan Society for Precision Engineering), among others. He has received awards for his papers from the Japan Society for Precision Engineering and the Japan Society for the Promotion of Machine Tool Engineering, and awards for meritorious achievements from the design engineering division of the ASME.

\section{Kazuhiro Izui}

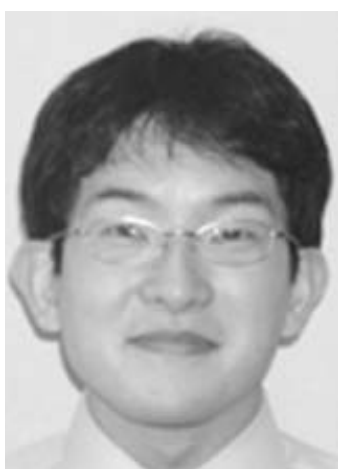

Kazuhiro Izui earned his Bachelor of Engineering Degree from Kyoto University and his Master Degree in Precision Engineering from Kyoto University in 1998. He is now a Research Associate in the Graduate School of Engineering at Kyoto University. His research topics are concurrent design optimization techniques using evolutional computing and product development management systems involving collaborative design optimization.

\section{Shusaku Kida}

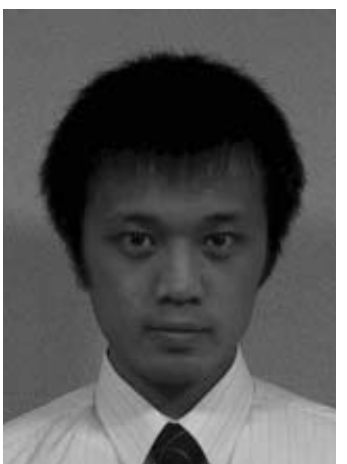

Shusaku Kida earned his Bachelor of Engineering Degree from Kyoto University in 2002. He is a master course student in the School of Information Science at the Nara Institute of Science and Technology. Currently, his research topics are focused on image processing methods. 